\section{Hybrid Electric Systems: Goals, Strategies, and Top Accomplishments}

Transitioning America's vehicle fleet to electric-drive vehicles could reduce U.S. foreign oil dependence by more than $60 \%$ and greenhouse gas emissions by $40 \%$ while increasing the nation's economic security. But electric-drive vehicles aren't new. At the start of the 20th Century, electric cars with lead-acid batteries held much of the U.S. market. Their popularity waned as the interest in cars with internal combustion engines (ICEs) rose, owing to the ICE vehicle's longer driving range, declining petroleum costs, and the advent of the electric starter and manufacturing assembly line.

Today, electric-drive technologies are back and ready to compete with-and complement - the ubiquitous ICE technology. Advances in electric-drive technologies have enabled commercialization of hybrid electric vehicles (HEVs), which combine an ICE with batteries and an electric motor to provide high fuel economy and a long driving range. Continued advances are spawning high-performance plug-in HEVs (PHEVs) - with small ICEs and large, grid-chargeable batteries that enable 10-40 mile all-electric driving rangesand electric vehicles (EVs) that don't use an ICE at all.

\section{Goals}

- By 2014, reduce the production cost of market-ready, highenergy, high-power batteries by $70 \%$ from 2009 costs.

- By 2015, reduce the cost of a market-ready advanced electric propulsion system by at least $35 \%$.

The Hybrid Electric Systems subprogram of the U.S Department of Energy's (DOE) Vehicle Technologies Program
(VTP) is spearheading the advances in energy storage and electric-drive technologies needed for the new generation of electric-drive vehicles, from battery materials R\&D to integration of the vehicle drivetrain. VTP goals, strategies, and major accomplishments are described below.

\section{Goals}

A crucial step toward electrified transportation is large-scale production of PHEVs that are cost-competitive with conventional ICE vehicles. VTP goals focus on the $\mathrm{R} \& \mathrm{D}$ required to help this happen: reducing the production cost of marketready, high-energy, high-power batteries by $70 \%$ by 2014 (compared with 2009 costs); and reducing the cost of a marketready advanced electric propulsion system by at least $35 \%$ by 2015 . These goals are complemented by technical targets - developed through system- and vehicle-level simulations - and are designed to ensure performance, safety, and reliability comparable to today's vehicles.

\section{Strategies}

VTP strategies for enabling largescale production of cost-competitive PHEVs are based on three complementary component- and system-level technology pathways:

1) Reduce the cost of electrochemical energy storage by developing lithium-ion batteries and other advanced energy storage technologies that afford higher energy densities without sacrificing safety and performance.

2) Enable the use of advanced energy storage technologies in vehicle systems by developing low-cost advanced power electronics and electric motor components.

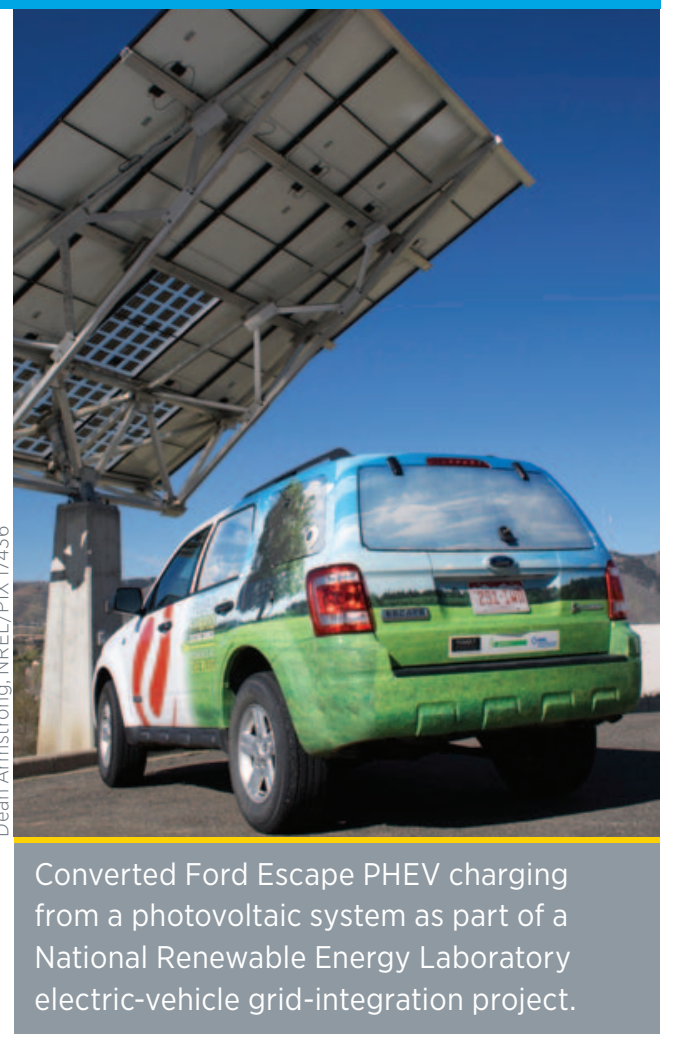

3) Develop and validate models and simulation tools to predict the performance, fuel economy, and emissions of advanced vehicle systems, in conjunction with laboratory and field testing, to benchmark and validate the operational characteristics of advanced electric-drive vehicles.

VTP collaborates with industry, universities, and national laboratories to conduct fundamental research on next-generation energy storage and electric-drive technologies as well as applied research that addresses the cross-cutting barriers inhibiting the commercialization of advanced electric-drive vehicles. Technology development conducted in collaboration with industry partnersincluding vehicle manufacturers and component developers and suppliersenables rapid integration of new technologies into commercial vehicles.

Finally, VTP helped direct $\$ 2.4$ billion in funds from the American Recovery and Reinvestment Act of 2009 to 48 new component manufacturing and vehicle 
Chevy Volt PHEV

deployment projects, including battery and power electronics manufacturing facilities, PHEV and EV demonstration projects, and education projects. This bolstering of U.S. capacity to manufacture and deploy electric-drive technologies is a critical cost-reduction strategy.

\section{Top Accomplishments}

VTP has achieved major advances related to energy storage, power electronics and electric machines, and vehicle and systems simulation and testing for HEVs and PHEVs. These include the ongoing development of innovative technologies and the demonstration and widespread deployment of advanced technologies in commercially available vehicles. The following are some of the top accomplishments to date.

\section{Energy Storage}

\section{Developed Today's HEV Batteries}

VTP-sponsored R\&D resulted in the state-of-the-art nickel metal hydride (NiMH) batteries used in all of today's HEVs. This support began in 1992 and was instrumental in the development of NiMH technology by Energy Conversion Devices (ECD) and Saft America. ECD has licensing agreements with Sanyo (which supplies HEV batteries to Ford and Honda) and Panasonic (which supplies HEV batteries to Toyota).

\section{Powered the Chevy Volt PHEV}

General Motors (GM) chose lithiummanganese-spinel battery technologydeveloped by Compact Power/LG Chem with VTP support - for its Chevy Volt Extended Range Electric Vehicle. The Volt is expected to be the first commercially available PHEV when it is released in late 2010. It is designed to travel 40 miles on a single battery charge.

\section{Developed the First Commercial Lithium-Ion HEV Battery}

VTP supported Johnson Controls-Saft (JCS) in developing the first lithium-ion battery incorporated into a production HEV. JCS supplies lithium-ion batteries

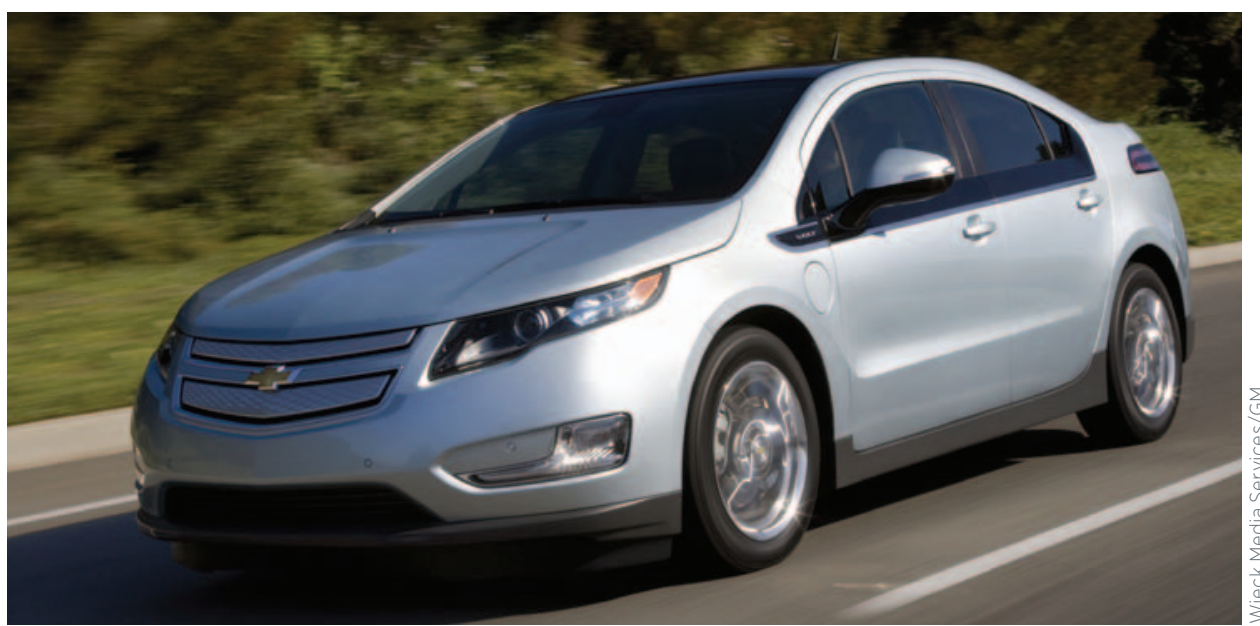

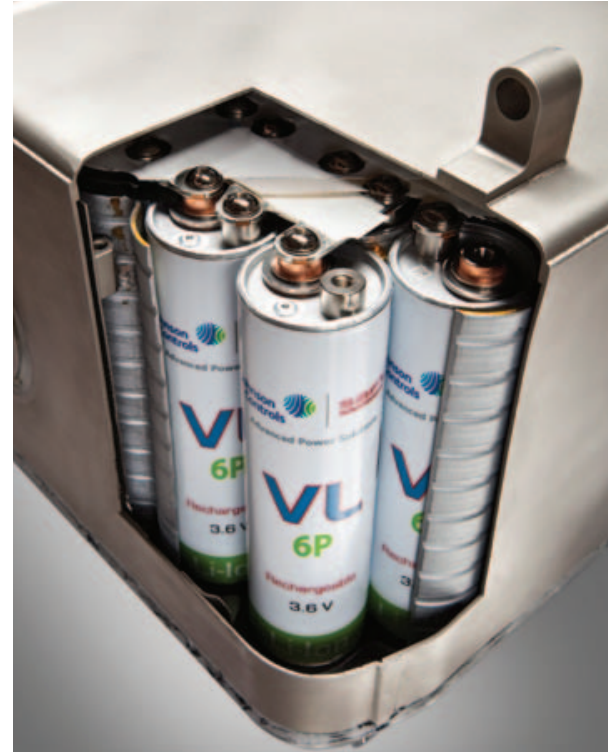

Johnson Controls-Saft lithium-ion battery

to Mercedes for its S400 BlueHybrid, introduced to the market in October 2009. This compact battery system performs better than the NiMH batteries used in other HEVs while achieving exceptional reliability, long service life, and excellent safety. JCS also will supply lithium-ion batteries to BMW for its 7-Series ActiveHybrid model.

\section{Developed Advanced Lithium Battery Materials}

VTP supported Argonne National Laboratory (ANL) in developing a diverse portfolio of patented lithium-ion battery technologies. For example, ANL developed a new lithium-ion cathode material that will result in batteries with better performance, service life, and safety than those using the cobalt-based

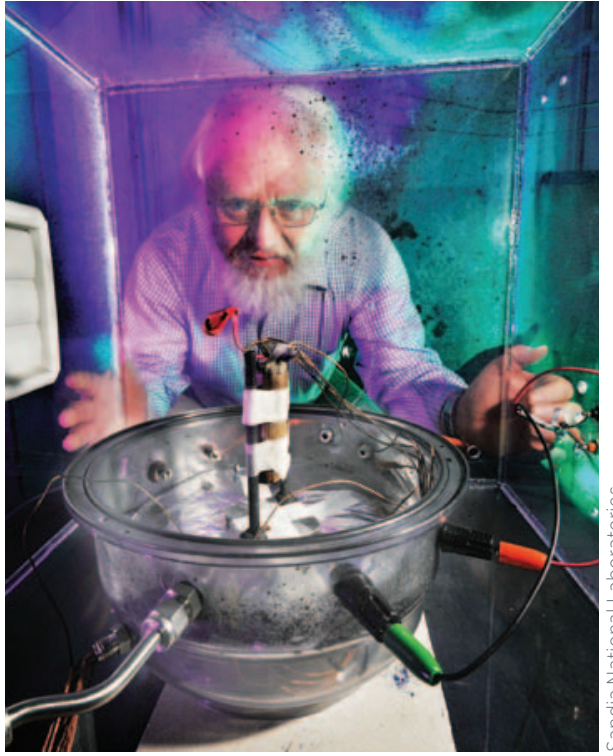

Investigating the behavior and safety of lithium-ion batteries under abuse conditions at Sandia National Laboratories.

cathode material that has dominated the market for nearly two decades. The patented cathode material - a lithium-rich nickel manganese oxide - was licensed to BASF and Toda Kogyo Corporation.

Another example is VTP's support of novel polymer electrolyte technology developed by Lawrence Berkeley National Laboratory (LBNL). Lithium-metal batteries with polymer electrolytes have a higher energy density than conventional lithium-ion batteries but suffer from poor ionic conductivity. The LBNL solid polymer technology enables high conductivity, longer life, and improved safety by replacing the traditional flammable liquid electrolytes. This patented electrolyte technology has been licensed to Seeo. 

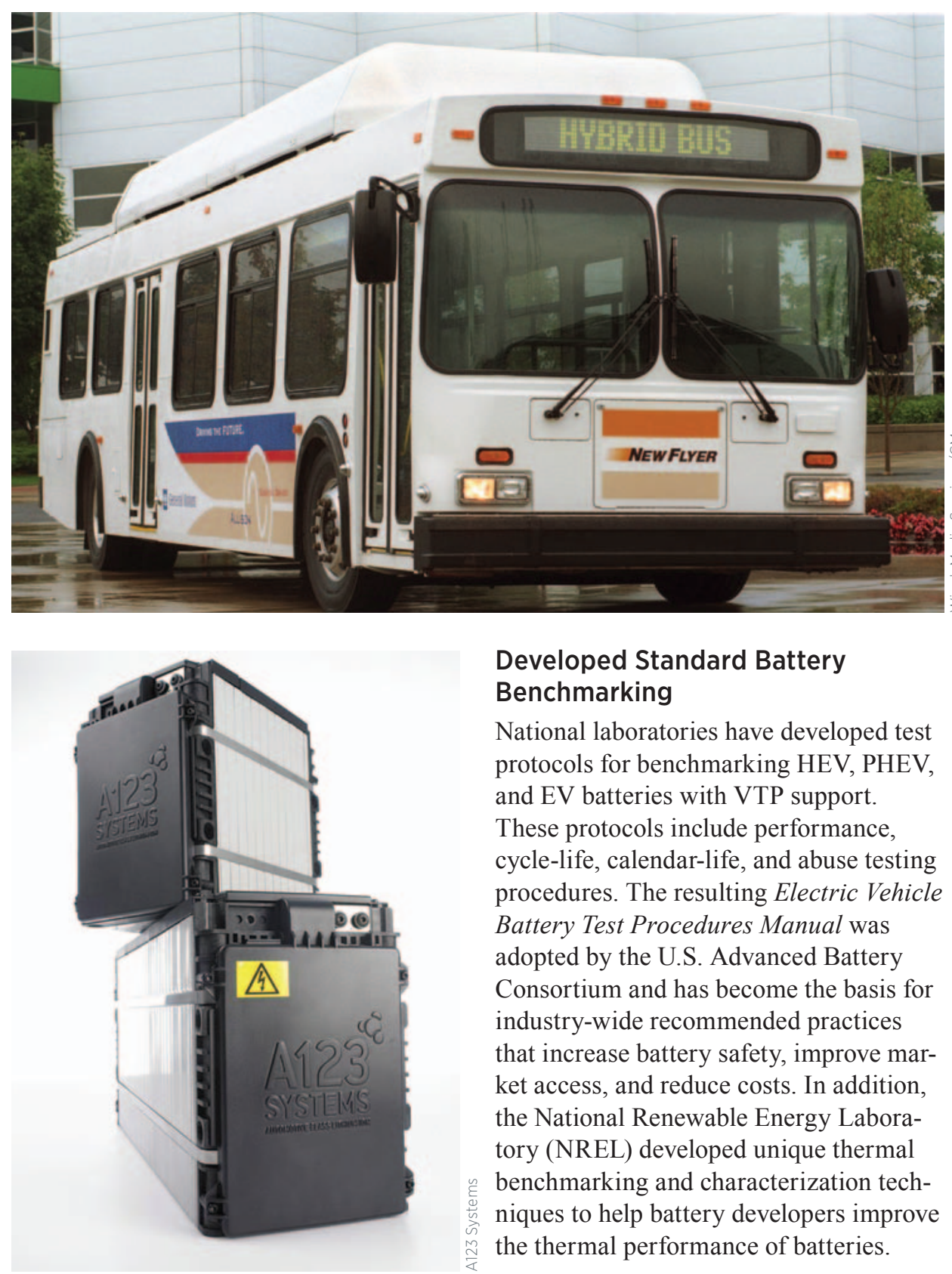

A123 Systems lithium-ion batteries

\section{Nurtured a New U.S. Battery Company}

Over the past decade, VTP support enabled A123 Systems to develop advanced lithium-ion batteries based on its nanoscale lithium-iron-phosphate cathode material. This material provides better safety, excellent cycle and calendar life, and higher rates of charge and discharge compared with conventional lithium-ion cathode materials. Through VTP-supported contracts, A123 Systems is beginning to commercialize lithium-ion battery systems for HEVs and PHEVs.

\section{Developed Standard Battery Benchmarking}

National laboratories have developed test protocols for benchmarking HEV, PHEV, and EV batteries with VTP support.

These protocols include performance, cycle-life, calendar-life, and abuse testing procedures. The resulting Electric Vehicle Battery Test Procedures Manual was adopted by the U.S. Advanced Battery Consortium and has become the basis for industry-wide recommended practices that increase battery safety, improve market access, and reduce costs. In addition, the National Renewable Energy Laboratory (NREL) developed unique thermal benchmarking and characterization techniques to help battery developers improve the thermal performance of batteries.

\section{Power Electronics and Electric Machines \\ Boosted HEV Fuel Economy}

In collaboration with GM and Allison Transmission, VTP sponsored the development of an advanced two-mode hybrid system for transit buses. A two-mode hybrid system enables high performance and fuel economy over a range of conditions by allowing the vehicle to function optimally in two separate modes: low speed/light load and high speed/heavy load. Two-mode hybrid buses achieve up to $50 \%$ better fuel economy than conventional buses. Recently, the same

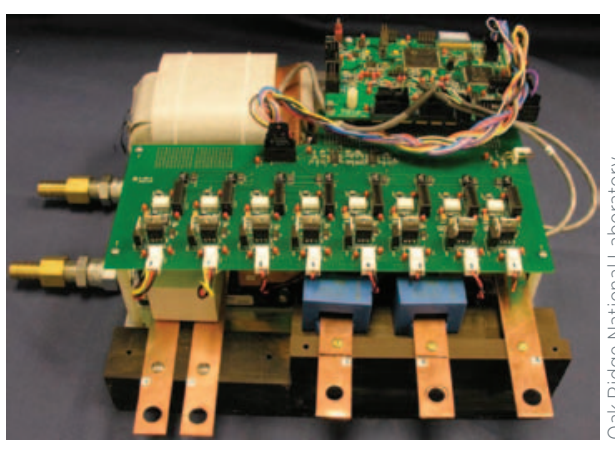

Current source inverter developed at Oak Ridge National Laboratory

GM-Allison two-mode hybrid bus

technology was applied to passenger HEVs, including the Chevy Tahoe Hybrid, GMC Sierra Hybrid, BMW ActiveHybrid X6, and Mercedes ML450 Hybrid.

\section{Accelerated Advanced Inverter Technology}

Electric-drive vehicles use an inverter to transform direct-current electricity from the batteries to the alternating current needed to power the electric motor. Because existing options were too large and expensive for certain advanced vehicle applications, VTP sponsored Semikron in developing an inverter with improved technical and cost characteristics. Components of the inverter were incorporated into vehicles used in Project Driveway, a GM program to test the real-world performance of fuel cell vehicles. Project Driveway vehicles recently surpassed 1 million miles of operation. VTP support was credited with accelerating the implementation of the new inverter technology in automotive applications.

\section{Collaborated to Stimulate Electric- Drive R\&D}

Collaboration between VTP and the U.S. Council for Automotive Research (USCAR) has stimulated domestic R\&D on electric-drive components and systems. Successes include new materials and packaging improvements that increase power density in automotive power electronics by $45 \%$ while improving their reliability and reducing their cost by $40 \%$. New advanced motors use bonded magnetic materials and less total magnetic material, which reduces 
manufacturing cost and energy use while enabling high-temperature operation. Research partners are also pursuing "leapfrog" technologies - which could push the state-of-the-art forward quickly and substantially - such as wide-bandgap power semiconductors that increase overall system efficiency and reliability.

\section{Vehicle and Systems Simulation and Testing Developed World-Class Modeling Tools}

With VTP support, ANL developed the Powertrain Systems Analysis Toolkit (PSAT) in 1999. PSAT enables dynamic analysis of vehicle performance and efficiency to support detailed design, hardware development, and validation of vehicle components and systems. In 2004, PSAT was selected as the primary vehicle model for all VTP activities. Today, it is used by more than 130 companiesincluding many major automakersand 700 users worldwide. It received an R\&D 100 Award in 2004 and a Federal Laboratory Consortium Technology Transfer Award in 2007.

In 2009, ANL began collaborating with the automotive industry to take PSAT to the next level. The result of this effort, Autonomie, was released in 2010. Autonomie will significantly expand VTP's component and vehicle modeling, testing, and evaluation capabilities. Among other uses, it will guide DOE-sponsored R\&D toward technologies with the greatest potential impact on vehicle fuel efficiency.

\section{Evaluated Next-Generation Vehicle Designs}

With VTP support, ANL and NREL apply PSAT and other tools to evaluate advanced technologies in a virtual environment before they are integrated into production vehicles. In 2010, NREL analyzed numerous hybrid architectures, battery and component sizes, and control strategies to evaluate design trade-offs affecting performance, fuel economy, cost, and marketability for light-, medium-, and heavyduty vehicles. Applied analyses such as these will help VTP and industry identify, which technology platforms are best for reducing petroleum consumption while maintaining competitive performance and cost.

\section{Validated \\ Component and Vehicle Technologies}

VTP sponsors field and laboratory testing to validate electric-drive component and model performance in a vehicle

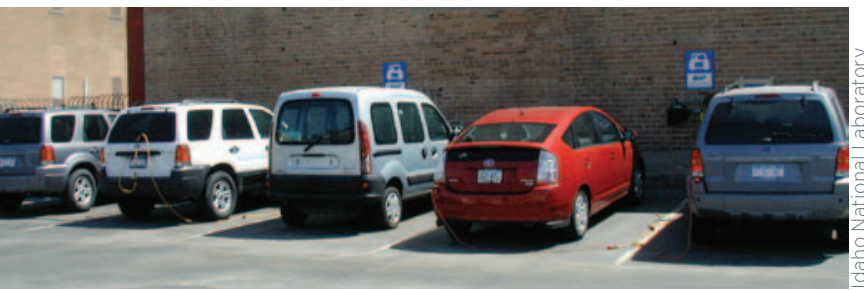

PHEVs charging as part of an Idaho National Laboratory field-testing project.

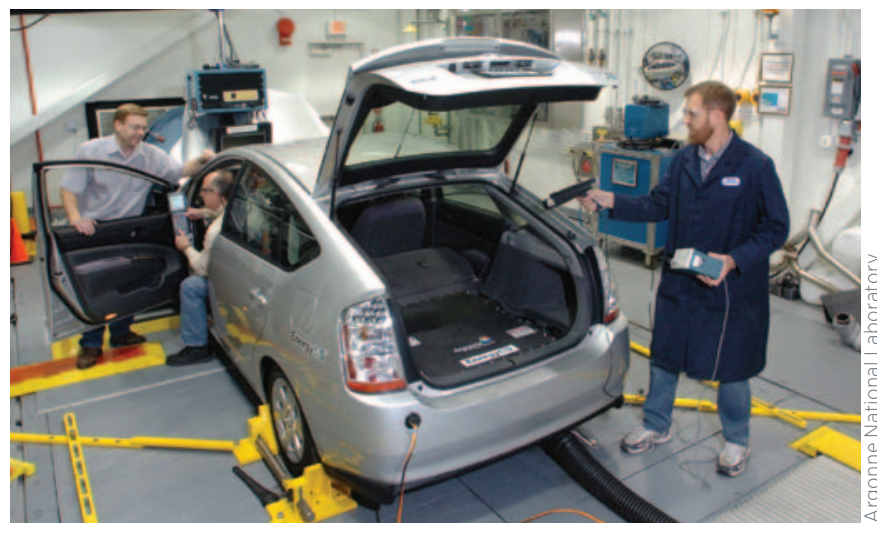

PHEV chassis dynamometer testing at Argonne National Laboratory's Advanced Powertrain Research Facility. systems context. ANL and Idaho National Laboratory have conducted reliability testing - including more than 1.5 million miles of on-road testing to date - on HEVs and PHEVs and are preparing for large-scale evaluation of EVs once they become available. NREL works with commercial and government fleets to evaluate the performance of medium- and heavy-duty advancedtechnology vehicles in service; one of the many technologies evaluated was the GM-Allison two-mode hybrid system previously discussed, deployed in transit buses. The data from these projects, which are made publicly available, are used by automakers to improve advanced-

\section{More Information}

For more information about the Hybrid Electric Systems subprogram, contact David Howell at david.howell@ ee.doe.gov. technology vehicles and by vehicle users to evaluate the viability of new technologies. The data also have been used to develop HEV fuel economy testing procedures such as SAE J1711.

\section{Electrifying the Future}

VTP has driven important advances in the electric-drive technologies needed to secure the nation's energy future. In collaboration with industry, universities, and national laboratories, VTP continues to develop the next-generation energy storage technologies, power electronics devices, additional electric-drive components, and modeling and testing capabilities needed to enable the complete integration of electric-drive systems. These $R \& D$ activities are designed to spur widespread deployment of HEVs, PHEVs, and EVs - and yield substantial reductions in petroleum consumption and greenhouse gas emissions.
Printed with a renewable-source ink on paper containing at least $50 \%$ wastepaper including $10 \%$ post consumer waste.
Prepared by the National Renewable Energy Laboratory (NREL) NREL is a national laboratory of the U.S. Department of Energy Office of Energy Efficiency and Renewable Energy Operated by the Alliance for Sustainable Energy, LLC.

DOE/GO-102010-3036 • June 2010 